\title{
A tropomyosin receptor kinase (TRK) family protein, NTRK2 is a potential predictive biomarker for lung adenocarcinoma
}

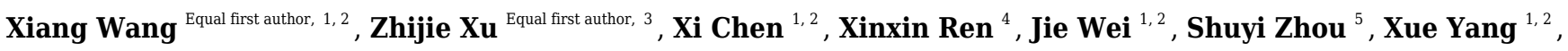 \\ Shuangshuang Zeng ${ }^{1,2}$, Long Qian ${ }^{1,2}$, Geting Wu ${ }^{3}$, Zhicheng Gong ${ }^{1,2}$, Yuanliang Yan ${ }^{\text {Corresp. } 1,2}$ \\ ${ }^{1}$ Department of Pharmacy, Xiangya Hospital, Central South University, Changsha, Hunan, China \\ 2 National Clinical Research Center for Geriatric Disorders, Xiangya Hospital, Central South University, Changsha, Hunan, China \\ 3 Department of Pathology, Xiangya Hospital, Central South University, Changsha, Hunan, China \\ 4 Center for Molecular Medicine, Xiangya Hospital, Key Laboratory of Molecular Radiation Oncology of Hunan Province, Central South University, \\ Changsha, Hunan, China \\ 5 Department of General Surgery, Hunan Provincial People's Hospital Xingsha Branch (People's Hospital of Changsha County), Changsha, Hunan, China \\ Corresponding Author: Yuanliang Yan \\ Email address: yanyuanliang@csu.edu.cn
}

Neurotrophic receptor tyrosine kinase 2 (NTRK2) is a member of the tropomyosin receptor kinase (TRK) family associated with the tumor development. However, the detailed function of NTRK2 in lung cancer, especially in lung adenocarcinoma (LUAD), is still not fully understood. Here, we investigated the effects of NTRK2 on LUAD biology. Through analyzing bioinformatics data derived from several databases, such as Oncomine, Gene Expression Profiling Interactive Analysis (GEPIA) and UALCAN, we found that NTRK2 expression was significantly decreased in LUAD tissues. Clinical data acquired from Wanderer database, which is linked to The Cancer Genome Atlas (TCGA) database, demonstrated that the expression and methylation site of NTRK2 were significantly related to the clinical characteristics and prognosis of LUAD. Furthermore, NTRK2 expression was increased remarkably after treatment with the protein kinase B (AKT) inhibitor MK2206 and the anticancer agent actinomycin D. Functional enrichment analysis of NTRK2-associated coexpression genes was further conducted. Together, our results suggested that downregulated NTRK2 might be used in the diagnostic and prognostic evaluation of LUAD patients, or as a potential therapeutic target for the treatment of LUAD. 
1

2

3

4

5

6

$7{ }^{2}$ National Clinical Research Center for Geriatric Disorders, Xiangya Hospital, Central South

8 University, Changsha 410008, Hunan, China.

\section{A tropomyosin receptor kinase (TRK) family protein, NTRK2 is a potential predictive biomarker for lung adenocarcinoma}

Xiang Wang ${ }^{1,2 \dagger}$, Zhijie $\mathrm{Xu}^{3 \dagger}$, Xi Chen ${ }^{1,2}$, Xinxin Ren ${ }^{4}$, Jie Wei ${ }^{1,2}$, Shuyi Zhou ${ }^{5}$, Xue Yang ${ }^{1,2}$, Shuangshuang Zeng ${ }^{1,2}$, Long Qian ${ }^{1,2}$, Geting $\mathrm{Wu}^{3}$, Zhicheng Gong ${ }^{1,2}$, Yuanliang Yan ${ }^{1,2 *}$

1 Department of Pharmacy, Xiangya Hospital, Central South University, Changsha 410008, Hunan, China.

3 Department of Pathology, Xiangya Hospital, Central South University, Changsha 410008, Hunan, China.

${ }^{4}$ Center for Molecular Medicine, Xiangya Hospital, Key Laboratory of Molecular Radiation 2 Oncology of Hunan Province, Central South University, Changsha 410008, Hunan, China.

${ }^{5}$ Department of General Surgery, Hunan Provincial People's Hospital Xingsha Branch (People's Hospital of Changsha County), Changsha 410008, Hunan, China.

$\uparrow$ These authors contributed equally to this work.

* Corresponding author: Yuanliang Yan; Email: yanyuanliang@csu.edu.cn 
Abstract: Neurotrophic receptor tyrosine kinase 2 (NTRK2) is a member of the tropomyosin receptor kinase (TRK) family associated with the tumor development. However, the detailed function of NTRK2 in lung cancer, especially in lung adenocarcinoma (LUAD), is still not fully understood. Here, we investigated the effects of NTRK2 on LUAD biology. Through analyzing bioinformatics data derived from several databases, such as Oncomine, Gene Expression Profiling Interactive Analysis (GEPIA) and UALCAN, we found that NTRK2 expression was significantly decreased in LUAD tissues. Clinical data acquired from Wanderer database, which is linked to The Cancer Genome Atlas (TCGA) database, demonstrated that the expression and methylation site of NTRK2 were significantly related to the clinical characteristics and prognosis of LUAD. Furthermore, NTRK2 expression was increased remarkably after treatment with the protein kinase B (AKT) inhibitor MK2206 and the anticancer agent actinomycin D. Functional enrichment analysis of NTRK2-associated coexpression genes was further conducted. Together, our results suggested that downregulated NTRK2 might be used in the diagnostic and prognostic evaluation of LUAD patients, or as a potential therapeutic target for the treatment of LUAD.

\section{Introduction}

Lung adenocarcinoma (LUAD) is the most frequent subtype of lung cancer, with incidence and mortality rates rising in both Western and Asian countries (Yan et al. 2019). Because of late diagnoses, the 5-year overall survival rate LUAD varies from 4 to $17 \%$ in line with the differences of stage and region, which is still very poor (Yan et al. 2018). At present, there is still no effective early diagnosis method for patients to receive timely treatment (Zheng et al. 2018). Therefore, it is necessary to search for novel target molecules for improving the early diagnosis and treatment of LUAD.

Previous studies have found a strong link between neurotrophic receptor tyrosine kinase 2 (NTRK2) and psychiatric disorders, such as schizophrenia (Spalek et al. 2016). Recent research advancement in the field revealed the relationship between NTRK2 and cancer biology. According to the ceRNA network, Gao. et al. found that NTRK2 is related to the prognosis of 
55

56

invasive breast cancer (Gao et al. 2019). Through constructing the coexpression modules by WGCNA, NTRK2 was proposed to play a key role in the recurrence of uveal melanoma (Wan et al. 2018). Ni. et al. demonstrated that activated NTRK2 alleles, especially the human tumorassociated QKI-NTRK2 fusion, could function together with Ink4a/Arf loss to promote astrocytoma formation (Ni et al. 2017). Furthermore, a recent study found that the interaction between differentiated glioblastoma cells and stem-like tumor cells via BDNF-NTRK2-VGF paracrine signaling accelerates tumor growth (Wang et al. 2018b). Nevertheless, there were few investigations about the relationship between NTRK2 and lung cancer, particularly LUAD, so the effects and mechanisms of NTRK2 in LUAD require further research.

The purpose of our study was to evaluate the role and mechanism of NTRK2 in human LUAD. Through bioinformatics data analysis, NTRK2 was found to be significantly downregulated in LUAD tissues. In addition, the expression level and methylation site of NTRK2 were notably correlated with clinical characteristics and prognosis. Moreover, based on the two datasets GSE6400 and GSE54293 from Gene Expression Omnibus (GEO), we observed the high levels of NTRK2 in the anticancer treatment group, indicating that NTRK2 could be used as a biomarker in evaluating clinical efficacy. In addition, Gene Ontology enrichment (GO) and Kyoto Encyclopedia of Genes and Genomes (KEGG) (Kanehisa \& Goto 2000) analysis of NTRK2-associated coexpression genes further indicated that NTRK2 played an important part in LUAD treatment.

\section{Materials and Methods}

2.1 Data acquisition and reanalysis using different bioinformatics tools

The relevant bioinformatics data analysis of NTRK2 was obtained from several bioinformatics web resources, which were summarized in Table S1. And the flow diagram of NTRK2 screen has been showed in Figure S1.

Oncomine is a cancer microarray or high-throughput sequencing data-mining platform, from which we can get gene expression signatures in human cancer tissues and cells (Rhodes et 
82

83

84

al. 2004). The data in Oncomine could be also link into other public databases, such as GEO and The Cancer Genome Atlas (TCGA) (Hutter \& Zenklusen 2018). We conducted the comparison of NTRK2 expression across eight analyses between the LUAD and normal tissues. Additionally, Gene Expression Profiling Interactive Analysis (GEPIA) (Tang et al. 2017a), GE-mini (Tang et al. 2017b), Cancer RNA-Seq Nexus (CRN) (Li et al. 2016) and UALCAN (Chandrashekar et al. 2017), four additional cancer microarray or high-throughput sequencing data-mining databases, were employed to verify the results.

Wanderer is an interactive viewer, providing gene expression and DNA methylation data in human cancer (Diez-Villanueva et al. 2015), which enables us to screen for the possible methylation sites in the NTRK2 DNA sequence and to analyze the correlation between clinical characteristic of LUAD patients and NTRK2 expression and methylation sites. For the prognostic analysis, Kaplan-Meier Plotter, a tool that can be used to assess the effect of genes on survival (Wang et al. 2018a), was utilized to describe the relationship between NTRK2 expression level, overall survival time (OS) and post-progression survival time (PPS). Further, the association between NTRK2 expression and disease free survival (RFS) was completed through the GEPIA database.

Two datasets of the treatment-related transcriptome microarray, GSE6400 (Wang et al. 2007) and GSE54293 (Denisova et al. 2014), were acquired from the GEO database (Barrett \& Edgar 2008). Subsequently, the effects of NTRK2 expression on the chemotherapy for LUAD were analyzed.

The expression and methylation of NTRK2 correlation analysis was implemented by MethHC, which provided the information of DNA methylation and gene expression in human cancer (Huang et al. 2015). For the relevance between the disease prognosis and the methylation sites of NTRK2, MethSurv tool was employed (Modhukur et al. 2018).

Using the cBioportal web tool (Gao et al. 2013), genes coexpressed with NTRK2 in LUAD were downloaded. Then, the STRING database (Szklarczyk et al. 2017) and Cytoscape software (Reimand et al. 2019) were used to complete the protein-protein interaction (PPI) network of 
109

110

111

112

113

114

115

116

117

118

119

120

121

122

123

124

125

126

127

128

129

130

131

132

133

134

135

these coexpression genes. Then, we utilized the DAVID bioinformatics resource (Huang da et al. 2009) to conduct the GO and KEGG pathway analysis of NTRK2 coexpression genes in LUAD samples. The web tools of WebGestalt (Wang et al. 2017) and PATHVIEW (Luo et al. 2017) were used for building a graphic.

2.2 Statistical analyses

The statistical tests were performed using SPSS 12.0 software (IBM Analytics). The results were expressed as the mean $\pm \mathrm{SD}$. Student $\mathrm{t}$ test, one-way ANOVA and K independent samples test were performed when appropriate. $\mathrm{P}<0.05$ was considered statistically significant.

\section{Results}

3.1 NTRK2 is downregulated in LUAD tissues

The NTRK family consists of three members, NTRK1, NTRK2 and NTRK3. Through the bioinformatics analysis of databases, we evaluated the transcriptional levels of NTRK family members in LUAD. First, we used the Oncomine database to observe the expression of NTRK1, NTRK2 and NTRK3 in eight LUAD datasets (Beer et al. 2002; Bhattacharjee et al. 2001; Hou et al. 2010; Landi et al. 2008; Okayama et al. 2012; Selamat et al. 2012; Stearman et al. 2005; Su et al. 2007). The results showed that NTRK2 had significantly lower expression in LUAD through the comparison among nine datasets, whereas NTRK1 and NTRK3 showed no statistical significance (Figure 1A). Therefore, NTRK2 was chosen as the research target. To verify the trend, we examined the NTRK2 expression in LUAD by GEPIA and GE-mini, and we discovered the NTRK2 expression was clearly reduced in LUAD compared with the normal tissues (Figure 1B-C). In addition, the heatmap from CRN database further indicated the low expression of NTRK2 in LUAD tissues (Figure 1D). Next, given some activated oncogenes, such as Erb-B2 receptor tyrosine kinase 2 (ERBB2) and MET, have been demonstrated the driver roles in LUAD (Cancer Genome Atlas Research 2014), we want to evaluate the association between NTRK2 and these oncogenes. The data from UALCAN revealed the significantly downregulated NTRK2 $(\mathrm{P}<0.01)$, upregulated ERBB2 $(\mathrm{P}<0.01)$ and upregulated MET $(\mathrm{P}<$ 
136

137

138

139

140

141

142

143

144

145

146

147

148

149

150

151

152

153

154

155

156

157

158

159

160

161

162

0.01) in LUAD tissues (Figure S2A). Spearman correlation analysis showed the negative association between the expression of NTRK2 and ERBB2 or MET (Figure S2B). Taken together, all of the above data suggested that the decreased expression of NTRK 2 contributed to LUAD tumorigenesis, supporting its tumor-inhibiting function in LUAD.

3.2 NTRK2 expression is associated with the clinical characteristics of LUAD patients

After determining the expression of NTRK2 in LUAD, we further analyzed the correlation between the NTRK2 expression level and the clinical characteristics of patients. Using the Wanderer database, we obtained a series of clinical data, and a summary of clinical characteristic parameters is provided in Table 1. As shown in this table, NTRK2 expression was significantly associated with gender $(\mathrm{P}=0.007)$, pathologic $T(\mathrm{P}=0.021)$, pathologic $\mathrm{M}(\mathrm{P}=0.006)$ and age $(\mathrm{P}=0.036)$. Then, the Kaplan-Meier Plotter tool was used to evaluate the effects of NTRK2 expression on OS and PPS, confirming that the downregulated of NTRK2 expression was significantly related to shorter OS $(\mathrm{P}=0.00029)$ (Figure $2 \mathrm{~A})$ and PPS $(\mathrm{P}=0.021)$ (Figure $2 \mathrm{~B})$. Furthermore, we found that low NTRK2 expression was associated with RFS $(\mathrm{P}=0.012)$ through using the GEPIA database (Figure 2C). In conclusion, NTRK2 could be as a potential biomarker both for diagnosis and prognosis.

3.3 The roles of NTRK2 in LUAD therapies

For the purpose of identifying the exact function of NTRK2 in LUAD chemotherapy, two treatment-related transcriptome microarray datasets, GSE6400 and GSE54293, were obtained from the GEO database. Previous studies have demonstrated that actinomycin D (Bai et al. 2019) and MK2206 (Dai et al. 2017) were two promising antitumor drugs. In the GSE6400 dataset, we discovered that the expression of NTRK2 was apparently higher in the actinomycin D treatment group than in the mannitol-control group $(\mathrm{P}=0.008)$ (Figure 3A). In addition, for the GSE54293 dataset, the AKT inhibitor MK2206 could enhance the NTRK2 expression levels significantly (P $=0.009)$ (Figure 3B). Collectively, the findings observed above suggested that NTRK2 might enhance the response of cancer cells to the chemotherapeutics.

3.4 The relationship between NTRK2 methylation and the clinical characteristics of LUAD 
163

164

165

166

167

168

169

170

171

172

173

174

175

176

177

178

179

180

181

182

183

184

185

186

187

188

189

patients

It is well-known that there is a negative correlation between DNA methylation and gene expression (Shi et al. 2017; Zhou et al. 2019). From the MethHC database, we observed that global NTRK2 methylation was significantly higher in LUAD samples compared with normal samples $(\mathrm{P}<0.005)$ (Figure 4A) and was negatively related to its expression $(\mathrm{P}=0.000)$ (Figure 4B), which gives further support for the low expression of NTRK2 in LUAD. Subsequently, the methylation site $\operatorname{cg} 03628748$ was screened out of the data $(\mathrm{P}=4.35 \mathrm{E}-12)$ (Table S2) acquired from the Wanderer database. Then, the relationship between $\operatorname{cg} 03628748$ and the clinical characteristics of LUAD patients was examined, and results showed that cg03628748 was significantly related to Kras mutation $(\mathrm{P}=0.038)$ and pathologic $\mathrm{T}(\mathrm{P}=0.000)$ (Table 2$)$. Moreover, there was a significant negative correlation between higher methylation value of $\operatorname{cg} 03628748$ and shorter OS in LUAD patients $(\mathrm{P}=0.034)$, which was analyzed by using the web tool of MethSurv (Figure 4C).

3.5 Functional enrichment analysis of NTRK2-associated coexpression genes

Using the cBioPortal database, 15146 genes that were notably coexpressed with NTRK2 in the LUAD samples were acquired. The volcano plot was established for exhibiting between the altered and unaltered NTRK2 expression group (Figure 5A). Next, we singled out 219 NTRK2associated codifferentially expressed genes (co-DEGs) with the criteria of $p$ value $<0.05$ and $\mid \log$ Ratio $\mid \geq 2$ (Table S3). Then, a PPT network of the co-DEGs was performed by using the STRING database and Cytoscape software (Figure 5B). For the purpose of comprehending the biological function for these co-DEGs, GO and KEGG analyses were conducted by WebGestalt and PATHVIEW web tools, respectively. The biological processes showed that these co-DEGs were mainly connected with biological regulation and metabolic processes (Figure 5C). For the analysis of cellular components, the coexpression genes were mainly localized on cell membranes (Figure 5D). For molecular function, protein binding was primarily enriched for these coexpression genes (Figure 5E). Furthermore, the KEGG pathway demonstrated that these genes were involved in the process of xenobiotics and drug metabolism by cytochrome P450 
190

191

192

193

194

195

196

197

198

199

200

201

202

203

204

205

206

207

208

209

210

211

212

213

214

215

216

(Table S4).

\section{Discussion}

This is the first study which presents comprehensive bioinformatic analysis of different public datasets that NTRK2 was identified as anti-oncogene in LUAD and could be used as a potential biomarker. Using the TCGA data from several databases, we found that NTRK2 expression was markedly decreased in LUAD tissues. The patients with downregulated NTRK2 expression and higher methylation values often had shorter OS, PPS and RFS.

NTRK2 belongs to the NTRK family and has been previously shown to have an important impact on the development of the nervous system (Cocco et al. 2018). However, recent studies have demonstrated the possible role of NTRK2 in the development of cancer. NTRK2 activation cooperates with PTEN deficiency through the activation of both the JAK-STAT3 and PI3KAKT pathways to induce aggressiveness, resistance to current therapies and poor prognosis of Tcell acute lymphoblastic leukemia (T-ALL) (Yuzugullu et al. 2016). Currently, NTRK fusion mutations have been reported to associate with oncogenic activation in various signaling pathways, such as AKT and MAPK, across multiple tumors (Stransky et al. 2014). Moreover, NTRK fusions were connected with poor survival in lung cancers (Rolfo \& Raez 2017). Interestingly, the reports seemed contrary to our results; this phenomenon might be explained by following reasons. First, it is known that different diseases or subtypes of tumors have diverse pathological states, which can change genes, functions. On the other hand, the structure, constitution and condition of genes may transformed, such as gene mutation, accompany with gene fusions. Furthermore, NTRK fusions are thought to occur at a low frequency across multiple tumor types (Vaishnavi et al. 2015). Additionally, although NTRK fusions were observed in rare cancer types, such as congenital infantile fibrosarcoma and secretory breast carcinoma, the occurrence in common cancers has been largely unexplored (Qaddoumi et al. 2016). Additionally, the difference in results might be on account of study designs or different patient populations, indicating international, multicenter randomized controlled, clinical research 
217 is needed for further study.

218 In the present study, GO and KEGG pathway analyses indicated that genes coexpressed 219 with NTRK2 were mainly enriched in the processes of xenobiotics and drug metabolism. 220 Moreover, NTRK2 expression was much higher in drug therapy groups in both the GSE6400 and 221 GSE54293 datasets. Therefore, up-regulating NTRK2 expression to promote drug metabolism 222 might be the mechanism that explains this phenomenon.

223 Nevertheless, there were several limitations to our study. First, the flow chart of analysis on 224 the roles of NTRK2 in LUAD tumorigenesis was not strong enough, and should be further

225

226

227

228

229

230

231

232

233

234

235

236

237

238

239

240

241

242

243

244

verified externally in diverse cohorts. Additionally, further validation of the roles of NTRK2 in multicenter clinical trials and prospective research is required. For the TCGA database, the included ethnicities were primarily white and black, and more studies are needed to confirm whether the findings are appropriate for other ethnic groups. Furthermore, more prognostic variables must be included to improve performance.

\section{Conclusion}

In conclusion, our study illustrated that NTRK2 was a putative cancer suppressor gene and could serve as a promising biomarker in tumorigenesis and treatment of LUAD patients. Furthermore, DNA hypermethylation has been demonstrated to be one of the mechanisms for the low-expressed NTRK2 in LADC. Understanding its detailed function and mechanisms in LUAD biological processes would provide promising insights for the prognostic and therapeutic value.

\section{Acknowledgments}

We thank Elsevier's English Language Editing Service for assistance with the language editing.

\section{References}

Bai L, Chen P, Xiang J, Sun J, and Lei X. 2019. Enantiomeric NMR discrimination of carboxylic acids using actinomycin D as a chiral solvating agent. Org Biomol Chem 17:1466-1470. 10.1039/c8ob03012j 
Barrett T, and Edgar R. 2008. Reannotation of array probes at NCBI's GEO database. Nat Methods 5:117. 10.1038/nmeth0208-117b

Beer DG, Kardia SL, Huang CC, Giordano TJ, Levin AM, Misek DE, Lin L, Chen G, Gharib TG, Thomas DG, Lizyness ML, Kuick R, Hayasaka S, Taylor JM, lannettoni MD, Orringer MB, and Hanash S. 2002. Gene-expression profiles predict survival of patients with lung adenocarcinoma. Nat Med 8:816-824. 10.1038/nm733

Bhattacharjee A, Richards WG, Staunton J, Li C, Monti S, Vasa P, Ladd C, Beheshti J, Bueno R, Gillette M, Loda M, Weber G, Mark EJ, Lander ES, Wong W, Johnson BE, Golub TR, Sugarbaker DJ, and Meyerson M. 2001. Classification of human lung carcinomas by mRNA expression profiling reveals distinct adenocarcinoma subclasses. Proc Natl Acad Sci U S A 98:13790-13795. 10.1073/pnas.191502998

Cancer Genome Atlas Research N. 2014. Comprehensive molecular profiling of lung adenocarcinoma. Nature 511:543-550. 10.1038/nature13385

Chandrashekar DS, Bashel B, Balasubramanya SAH, Creighton CJ, Ponce-Rodriguez I, Chakravarthi B, and Varambally S. 2017. UALCAN: A Portal for Facilitating Tumor Subgroup Gene Expression and Survival Analyses. Neoplasia 19:649-658. 10.1016/j.neo.2017.05.002

Cocco E, Scaltriti M, and Drilon A. 2018. NTRK fusion-positive cancers and TRK inhibitor therapy. Nat Rev Clin Oncol 15:731-747. 10.1038/s41571-018-0113-0

Dai S, Yan Y, Xu Z, Zeng S, Qian L, Huo L, Li X, Sun L, and Gong Z. 2017. SCD1 Confers Temozolomide Resistance to Human Glioma Cells via the Akt/GSK3beta/beta-Catenin Signaling Axis. Front Pharmacol 8:960. 10.3389/fphar.2017.00960

Denisova OV, Soderholm S, Virtanen S, Von Schantz C, Bychkov D, Vashchinkina E, Desloovere J, Tynell J, Ikonen N, Theisen LL, Nyman TA, Matikainen S, Kallioniemi O, Julkunen I, Muller CP, Saelens X, Verkhusha VV, and Kainov DE. 2014. Akt inhibitor MK2206 prevents influenza pH1N1 virus infection in vitro. Antimicrob Agents Chemother 58:3689-3696. 10.1128/AAC.02798-13

Diez-Villanueva A, Mallona I, and Peinado MA. 2015. Wanderer, an interactive viewer to explore DNA methylation and gene expression data in human cancer. Epigenetics Chromatin 8:22. 10.1186/s13072-015-0014-8

Gao C, Li H, Zhuang J, Zhang H, Wang K, Yang J, Liu C, Liu L, Zhou C, and Sun C. 2019. The construction and analysis of ceRNA networks in invasive breast cancer: a study based on The Cancer Genome Atlas. Cancer Manag Res 11:1-11. 10.2147/CMAR.S182521

Gao J, Aksoy BA, Dogrusoz U, Dresdner G, Gross B, Sumer SO, Sun Y, Jacobsen A, Sinha R, Larsson E, Cerami E, Sander C, and Schultz N. 2013. Integrative analysis of complex cancer genomics and clinical profiles using the cBioPortal. Sci Signal 6:pl1. 10.1126/scisignal.2004088

Hou J, Aerts J, den Hamer B, van ljcken W, den Bakker M, Riegman P, van der Leest C, van der Spek P, Foekens JA, Hoogsteden HC, Grosveld F, and Philipsen S. 2010. Gene expression-based classification of non-small cell lung carcinomas and survival prediction. PLoS One 5:e10312. 10.1371/journal.pone.0010312

Huang da W, Sherman BT, and Lempicki RA. 2009. Systematic and integrative analysis of large gene lists using DAVID bioinformatics resources. Nat Protoc 4:44-57. 10.1038/nprot.2008.211

Huang WY, Hsu SD, Huang HY, Sun YM, Chou CH, Weng SL, and Huang HD. 2015. MethHC: a database of DNA methylation and gene expression in human cancer. Nucleic Acids Res 43:D856-861. 10.1093/nar/gku1151

Hutter C, and Zenklusen JC. 2018. The Cancer Genome Atlas: Creating Lasting Value beyond Its Data. Cell 173:283285. 10.1016/j.cell.2018.03.042

Kanehisa M, and Goto S. 2000. KEGG: kyoto encyclopedia of genes and genomes. Nucleic Acids Res 28:27-30. 
Landi MT, Dracheva T, Rotunno M, Figueroa JD, Liu H, Dasgupta A, Mann FE, Fukuoka J, Hames M, Bergen AW, Murphy SE, Yang P, Pesatori AC, Consonni D, Bertazzi PA, Wacholder S, Shih JH, Caporaso NE, and Jen J. 2008. Gene expression signature of cigarette smoking and its role in lung adenocarcinoma development and survival. PLoS One 3:e1651. 10.1371/journal.pone.0001651

Li JR, Sun CH, Li W, Chao RF, Huang CC, Zhou XJ, and Liu CC. 2016. Cancer RNA-Seq Nexus: a database of phenotype-specific transcriptome profiling in cancer cells. Nucleic Acids Res 44:D944-951. 10.1093/nar/gkv1282

Luo W, Pant G, Bhavnasi YK, Blanchard SG, Jr., and Brouwer C. 2017. Pathview Web: user friendly pathway visualization and data integration. Nucleic Acids Res 45:W501-W508. 10.1093/nar/gkx372

Modhukur V, Iljasenko T, Metsalu T, Lokk K, Laisk-Podar T, and Vilo J. 2018. MethSurv: a web tool to perform multivariable survival analysis using DNA methylation data. Epigenomics 10:277-288. 10.2217/epi-20170118

Ni J, Xie S, Ramkissoon SH, Luu V, Sun Y, Bandopadhayay P, Beroukhim R, Roberts TM, Stiles CD, Segal RA, Ligon KL, Hahn WC, and Zhao JJ. 2017. Tyrosine receptor kinase B is a drug target in astrocytomas. Neuro Oncol 19:22-30. 10.1093/neuonc/now139

Okayama H, Kohno T, Ishii Y, Shimada Y, Shiraishi K, Iwakawa R, Furuta K, Tsuta K, Shibata T, Yamamoto S, Watanabe S, Sakamoto H, Kumamoto K, Takenoshita S, Gotoh N, Mizuno H, Sarai A, Kawano S, Yamaguchi R, Miyano S, and Yokota J. 2012. Identification of genes upregulated in ALK-positive and EGFR/KRAS/ALKnegative lung adenocarcinomas. Cancer Res 72:100-111. 10.1158/0008-5472.CAN-11-1403

Qaddoumi I, Orisme W, Wen J, Santiago T, Gupta K, Dalton JD, Tang B, Haupfear K, Punchihewa C, Easton J, Mulder H, Boggs K, Shao Y, Rusch M, Becksfort J, Gupta P, Wang S, Lee RP, Brat D, Peter Collins V, Dahiya S, George D, Konomos W, Kurian KM, McFadden K, Serafini LN, Nickols H, Perry A, Shurtleff S, Gajjar A, Boop FA, Klimo PD, Jr., Mardis ER, Wilson RK, Baker SJ, Zhang J, Wu G, Downing JR, Tatevossian RG, and Ellison DW. 2016. Genetic alterations in uncommon low-grade neuroepithelial tumors: BRAF, FGFR1, and MYB mutations occur at high frequency and align with morphology. Acta Neuropathol 131:833-845. 10.1007/s00401-016-1539-z

Reimand J, Isserlin R, Voisin V, Kucera M, Tannus-Lopes C, Rostamianfar A, Wadi L, Meyer M, Wong J, Xu C, Merico $D$, and Bader GD. 2019. Pathway enrichment analysis and visualization of omics data using g:Profiler, GSEA, Cytoscape and EnrichmentMap. Nat Protoc. 10.1038/s41596-018-0103-9

Rhodes DR, Yu J, Shanker K, Deshpande N, Varambally R, Ghosh D, Barrette T, Pandey A, and Chinnaiyan AM. 2004. ONCOMINE: a cancer microarray database and integrated data-mining platform. Neoplasia 6:1-6.

Rolfo C, and Raez L. 2017. New targets bring hope in squamous cell lung cancer: neurotrophic tyrosine kinase gene fusions. Lab Invest 97:1268-1270. 10.1038/labinvest.2017.91

Selamat SA, Chung BS, Girard L, Zhang W, Zhang Y, Campan M, Siegmund KD, Koss MN, Hagen JA, Lam WL, Lam S, Gazdar AF, and Laird-Offringa IA. 2012. Genome-scale analysis of DNA methylation in lung adenocarcinoma and integration with mRNA expression. Genome Res 22:1197-1211. 10.1101/gr.132662.111

Shi YX, Wang Y, Li X, Zhang W, Zhou HH, Yin JY, and Liu ZQ. 2017. Genome-wide DNA methylation profiling reveals novel epigenetic signatures in squamous cell lung cancer. BMC Genomics 18:901. 10.1186/s12864-0174223-3

Spalek K, Coynel D, Freytag V, Hartmann F, Heck A, Milnik A, de Quervain D, and Papassotiropoulos A. 2016. A 
common NTRK2 variant is associated with emotional arousal and brain white-matter integrity in healthy young subjects. Transl Psychiatry 6:e758. 10.1038/tp.2016.20

Stearman RS, Dwyer-Nield L, Zerbe L, Blaine SA, Chan Z, Bunn PA, Jr., Johnson GL, Hirsch FR, Merrick DT, Franklin WA, Baron AE, Keith RL, Nemenoff RA, Malkinson AM, and Geraci MW. 2005. Analysis of orthologous gene expression between human pulmonary adenocarcinoma and a carcinogen-induced murine model. Am J Pathol 167:1763-1775. 10.1016/S0002-9440(10)61257-6

Stransky N, Cerami E, Schalm S, Kim JL, and Lengauer C. 2014. The landscape of kinase fusions in cancer. Nat Commun 5:4846. 10.1038/ncomms5846

Su L, Chang CW, Wu YC, Chen KC, Lin CJ, Liang SC, Lin CH, Whang-Peng J, Hsu SL, Chen CH, and Huang CY. 2007. Selection of DDX5 as a novel internal control for Q-RT-PCR from microarray data using a block bootstrap re-sampling scheme. BMC Genomics 8:140. 10.1186/1471-2164-8-140

Szklarczyk D, Morris JH, Cook H, Kuhn M, Wyder S, Simonovic M, Santos A, Doncheva NT, Roth A, Bork P, Jensen L, and von Mering C. 2017. The STRING database in 2017: quality-controlled protein-protein association networks, made broadly accessible. Nucleic Acids Res 45:D362-D368. 10.1093/nar/gkw937

Tang Z, Li C, Kang B, Gao G, Li C, and Zhang Z. 2017a. GEPIA: a web server for cancer and normal gene expression profiling and interactive analyses. Nucleic Acids Res 45:W98-W102. 10.1093/nar/gkx247

Tang Z, Li C, Zhang K, Yang M, and Hu X. 2017b. GE-mini: a mobile APP for large-scale gene expression visualization. Bioinformatics 33:941-943. 10.1093/bioinformatics/btw775

Vaishnavi A, Le AT, and Doebele RC. 2015. TRKing down an old oncogene in a new era of targeted therapy. Cancer Discov 5:25-34. 10.1158/2159-8290.CD-14-0765

Wan Q, Tang J, Han Y, and Wang D. 2018. Co-expression modules construction by WGCNA and identify potential prognostic markers of uveal melanoma. Exp Eye Res 166:13-20. 10.1016/j.exer.2017.10.007

Wang J, Vasaikar S, Shi Z, Greer M, and Zhang B. 2017. WebGestalt 2017: a more comprehensive, powerful, flexible and interactive gene set enrichment analysis toolkit. Nucleic Acids Res 45:W130-W137. 10.1093/nar/gkx356

Wang X, Chen D, Gao J, Long H, Zha H, Zhang A, Shu C, Zhou L, Yang F, Zhu B, and Wu W. 2018a. Centromere protein $\mathrm{U}$ expression promotes non-small-cell lung cancer cell proliferation through FOXM1 and predicts poor survival. Cancer Manag Res 10:6971-6984. 10.2147/CMAR.S182852

Wang X, Prager BC, Wu Q, Kim LY, Gimple RC, Shi Y, Yang K, Morton AR, Zhou W, Zhu Z, Obara EAA, Miller TE, Song A, Lai S, Hubert CG, Jin X, Huang Z, Fang X, Dixit D, Tao W, Zhai K, Chen C, Dong Z, Zhang G, Dombrowski SM, Hamerlik P, Mack SC, Bao S, and Rich JN. 2018b. Reciprocal Signaling between Glioblastoma Stem Cells and Differentiated Tumor Cells Promotes Malignant Progression. Cell Stem Cell 22:514-528 e515. 10.1016/j.stem.2018.03.011

Wang Z, Lecane PS, Thiemann P, Fan Q, Cortez C, Ma X, Tonev D, Miles D, Naumovski L, Miller RA, Magda D, Cho DG, Sessler JL, Pike BL, Yeligar SM, Karaman MW, and Hacia JG. 2007. Synthesis and biologic properties of hydrophilic sapphyrins, a new class of tumor-selective inhibitors of gene expression. Mol Cancer 6:9. 10.1186/1476-4598-6-9

Yan Y, Xu Z, Hu X, Qian L, Li Z, Zhou Y, Dai S, Zeng S, and Gong Z. 2018. SNCA Is a Functionally Low-Expressed Gene in Lung Adenocarcinoma. Genes (Basel) 9. 10.3390/genes9010016

Yan Y, Xu Z, Qian L, Zeng S, Zhou Y, Chen X, Wei J, and Gong Z. 2019. Identification of CAV1 and DCN as potential predictive biomarkers for lung adenocarcinoma. Am J Physiol Lung Cell Mol Physiol. 
10.1152/ajplung.00364.2018

Yuzugullu H, Von T, Thorpe LM, Walker SR, Roberts TM, Frank DA, and Zhao JJ. 2016. NTRK2 activation cooperates with PTEN deficiency in T-ALL through activation of both the PI3K-AKT and JAK-STAT3 pathways. Cell Discov 2:16030. 10.1038/celldisc.2016.30

Zheng H, Zhan Y, Liu S, Lu J, Luo J, Feng J, and Fan S. 2018. The roles of tumor-derived exosomes in non-small cell lung cancer and their clinical implications. J Exp Clin Cancer Res 37:226. 10.1186/s13046-018-0901-5

Zhou S, Yan Y, Chen X, Wang X, Zeng S, Qian L, Wei J, Yang X, Zhou Y, Gong Z, and Xu Z. 2019. Roles of highly expressed PAICS in lung adenocarcinoma. Gene 692:1-8. 10.1016/j.gene.2018.12.064

\section{Figure Legends}

Figure 1. Analysis of NTRK2 expression levels in LUAD tissues. (A) The comparison of the messenger RNA (mRNA) expression of NTRK (NTRK1, NTRK2 and NTRK3) among eight datasets by comparing the surrounding normal lung tissues and LUAD. (B-D) The mRNA expression of NTRK2 was evaluated from the database GEPIA, GE-mini and CRN, respectively. Figure 2. The effects of NTRK2 expression on prognosis in LUAD patients. (A-B) The relationship between NTRK2 expression and OS and PPS, described by Kaplan-Meier Plotter. (C) The association between NTRK2 expression and RFS within the GEPIA database.

Figure 3. The influence of NTRK2 on the therapeutic response of LUAD patients. (A) The GSE6400 dataset acquired from the GEO database was employed to estimate the impacts of NTRK2 expression on LUAD therapy both in the actinomycin D treatment group and the mannitol-control group. (B) In the treatment-related microarray GSE54293 dataset, the influence of NTRK2 expression on AKT inhibitor MK2206 treatment was evaluated.

Figure 4. The relationship between NTRK2 methylation and the clinical characteristics of LUAD patients. (A) Global NTRK2 methylation in LUAD samples compared with the normal samples analyzed by MethHC database. (B) The association between global NTRK2 methylation and its expression in LUAD samples using the MethHC database. (C) The impact of the methylation site $\operatorname{cg} 03628748$ in NTRK2 on OS in LUAD patients as analyzed by the MethSurv web tool.

Figure 5. Functional enrichment analysis of NTRK2-associated co-DEGs in LUAD. (A) The coexpression genes of NTRK2 were shown as volcano plot. (B) The PPI network of NTRK2- 
398 associated co-DEGs as completed by the STRING and Cytoscape software. (C-E) The GO 399 analysis of NTRK2 associated co-DEGs including biological processes, cellular components and 400 molecular function.

401

402 
Figure 1

Analysis of NTRK2 expression levels in LUAD tissues.

(A) The comparison of the messenger RNA (mRNA) expression of NTRK (NTRK1, NTRK2 and

NTRK3) among eight datasets by comparing the surrounding normal lung tissues and LUAD.

(B-D) The mRNA expression of NTRK2 was evaluated from the database GEPIA, GE-mini and

CRN, respectively.

\begin{tabular}{|c|c|c|c|c|c|c|c|c|c|c|}
\hline & & & & \multicolumn{7}{|c|}{ LUAD vs. Normal Lung } \\
\hline Median Rank & p-Value & Gene & & & & & & & & \\
\hline \multirow[t]{2}{*}{7456.0} & 0.140 & NTRK1 & & & & & & & & \\
\hline & & & 1 & 2 & 3 & 4 & 5 & 6 & 7 & 8 \\
\hline Median Rank & p-Value & Gene & & & & & & & & \\
\hline \multirow[t]{2}{*}{935.5} & $1.39 \mathrm{E}-5$ & NTRK2 & & & & & & & & \\
\hline & & & 1 & 2 & 3 & 4 & 6 & 6 & 7 & 8 \\
\hline Median Rank & p-Value & Gene & & & & & & & & \\
\hline \multirow[t]{2}{*}{1831.0} & 0.096 & NTRK3 & & & & & & & & \\
\hline & & & 1 & 2 & 3 & 4 & 6 & 6 & 7 & 8 \\
\hline
\end{tabular}

Legend

1. LUAD vs. Normal Lung Beer Lung, Nat Med, 2002

2. LUAD vs. Normal Lung Bhattacharjee Lung, Proc Natl Acad Sci USA, 2001

3. LUAD vs. Normal Lung Hou Lung, Plos One, 2010

4. LUAD vs. Normal Lung

Landi Lung, Plos One, 2008

$\begin{array}{llllllll}1 & 6 & 10 & 25 & 25 & 10 & 6 & 1\end{array}$

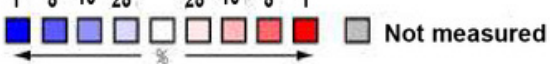

5. LUAD vs. Normal Lung Okayama Lung, Cancer Res, 2012

6. LUAD vs. Normal Lung Selamat Lung, Genome Res, 2012

7. LUAD vs. Normal Lung

Stearman Lung, Am J Pathol, 2005

8. LUAD vs. Normal Lung

Su Lung, BMC Genomics, 2007
B

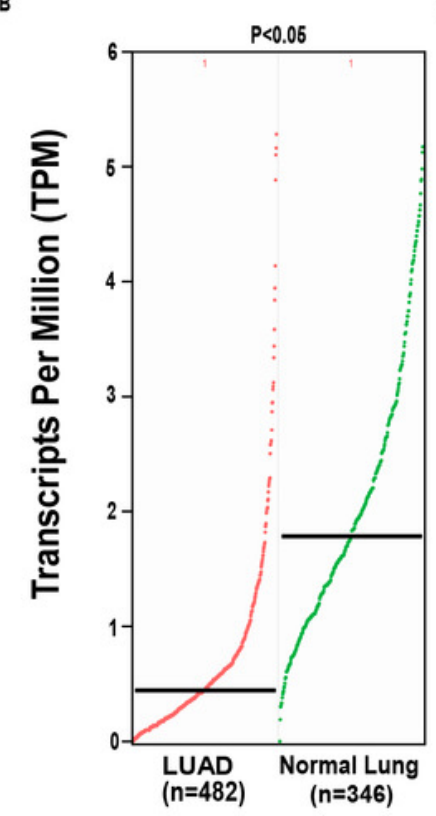

C

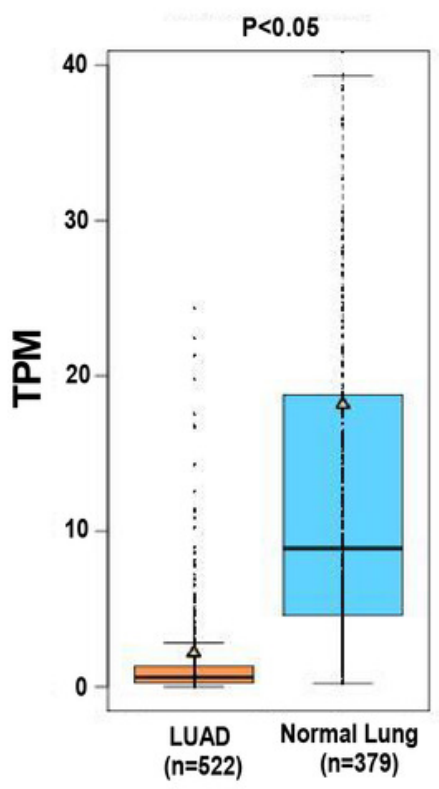

D

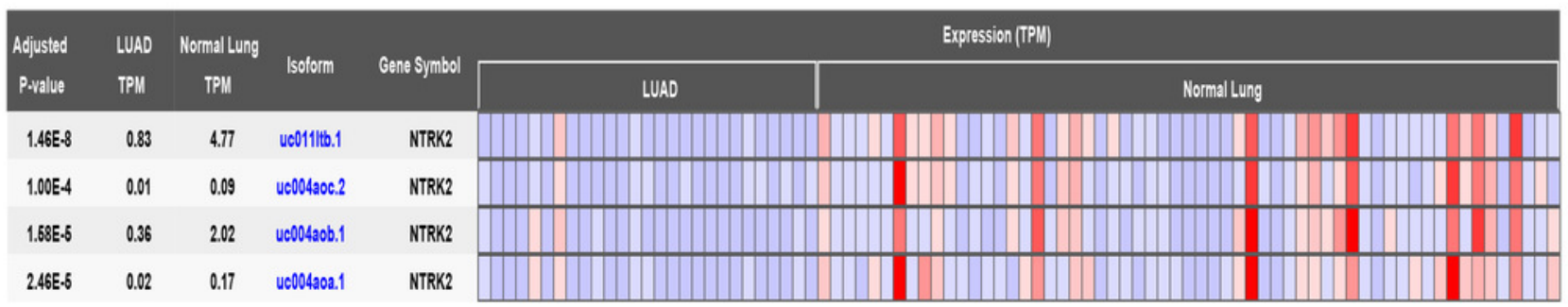


Figure 2

The effects of NTRK2 expression on prognosis in LUAD patients.

(A-B) The relationship between NTRK2 expression and OS and PPS, described by KaplanMeier Plotter. (C) The association between NTRK2 expression and RFS within the GEPIA database.

A

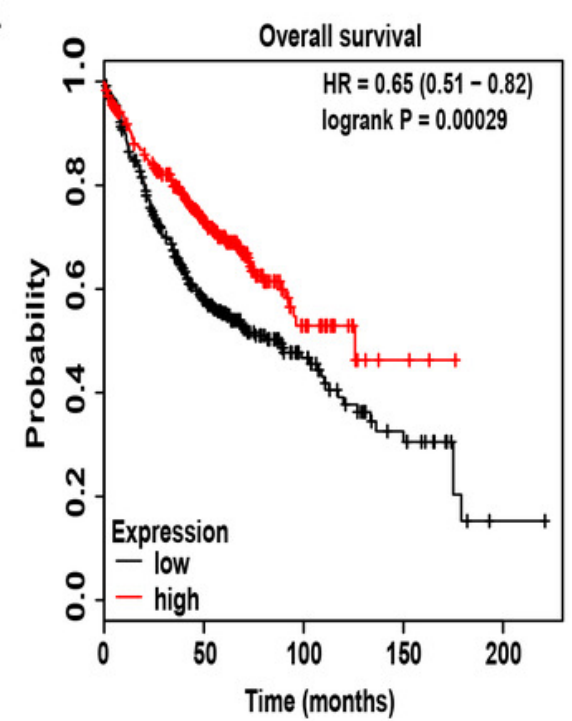

B

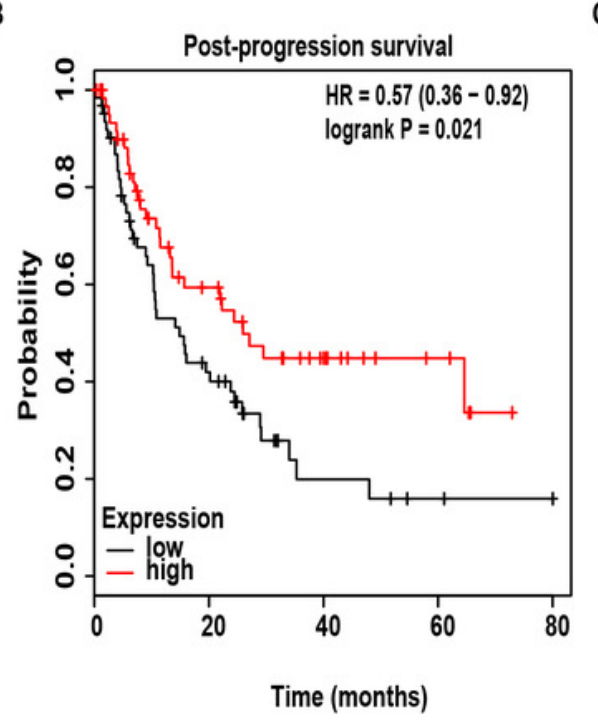

C

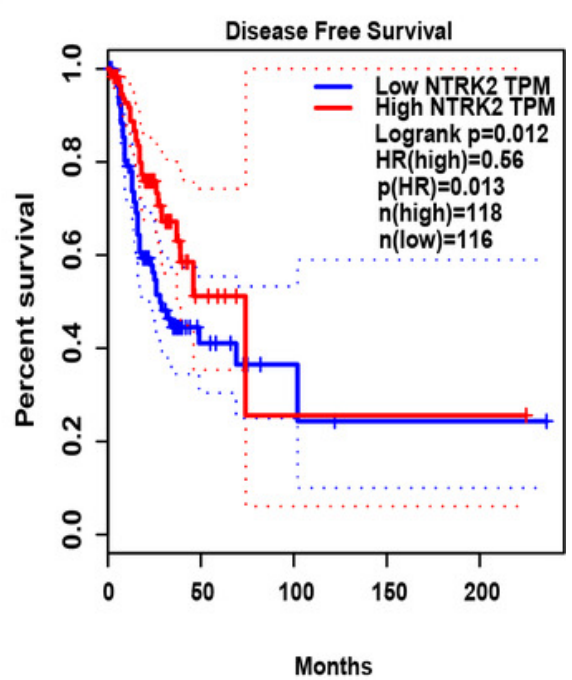


Figure 3

The influence of NTRK2 on the therapeutic response of LUAD patients.

(A) The GSE6400 dataset acquired from the GEO database was employed to estimate the impacts of NTRK2 expression on LUAD therapy both in the actinomycin D treatment group and the mannitol-control group. (B) In the treatment-related microarray GSE54293 dataset, the influence of NTRK2 expression on AKT inhibitor MK2206 treatment was evaluated.

A

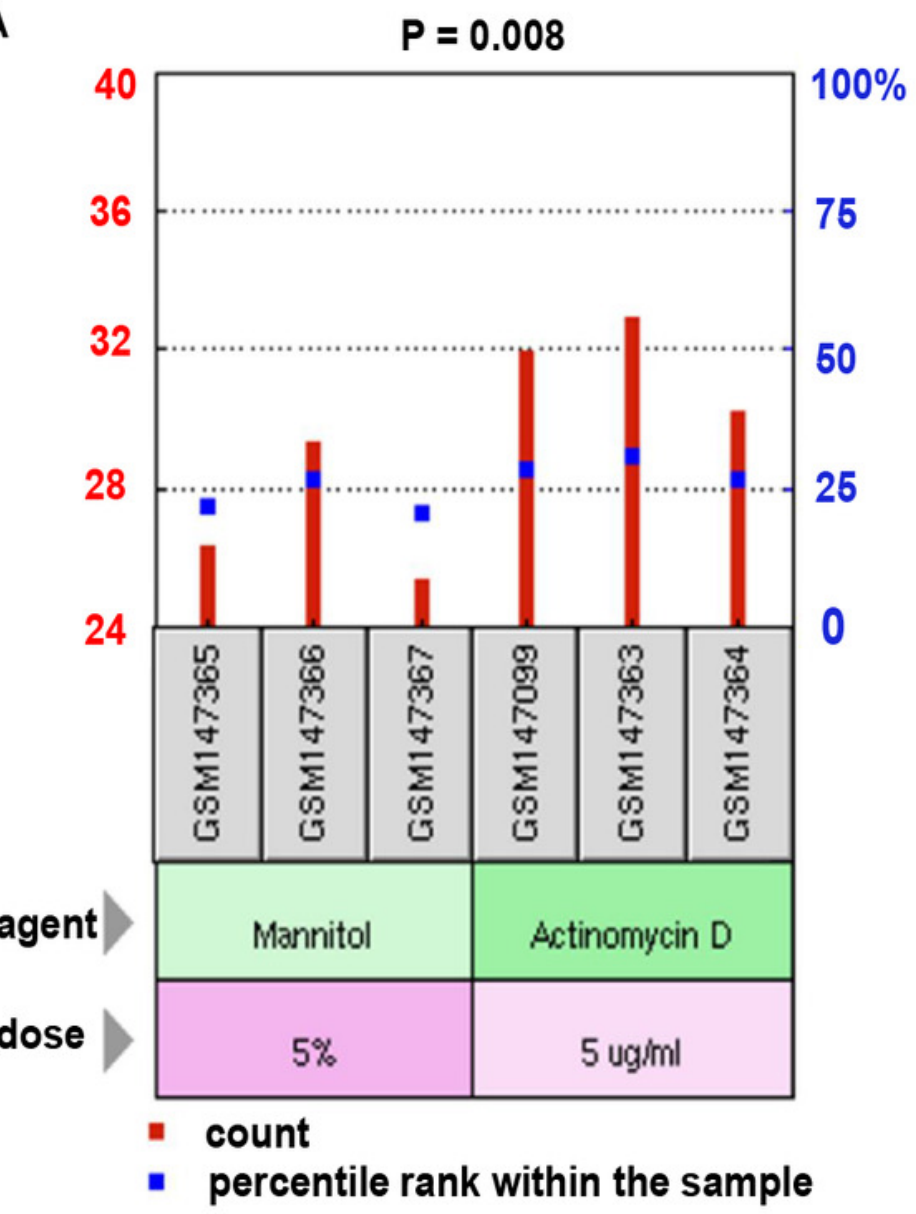

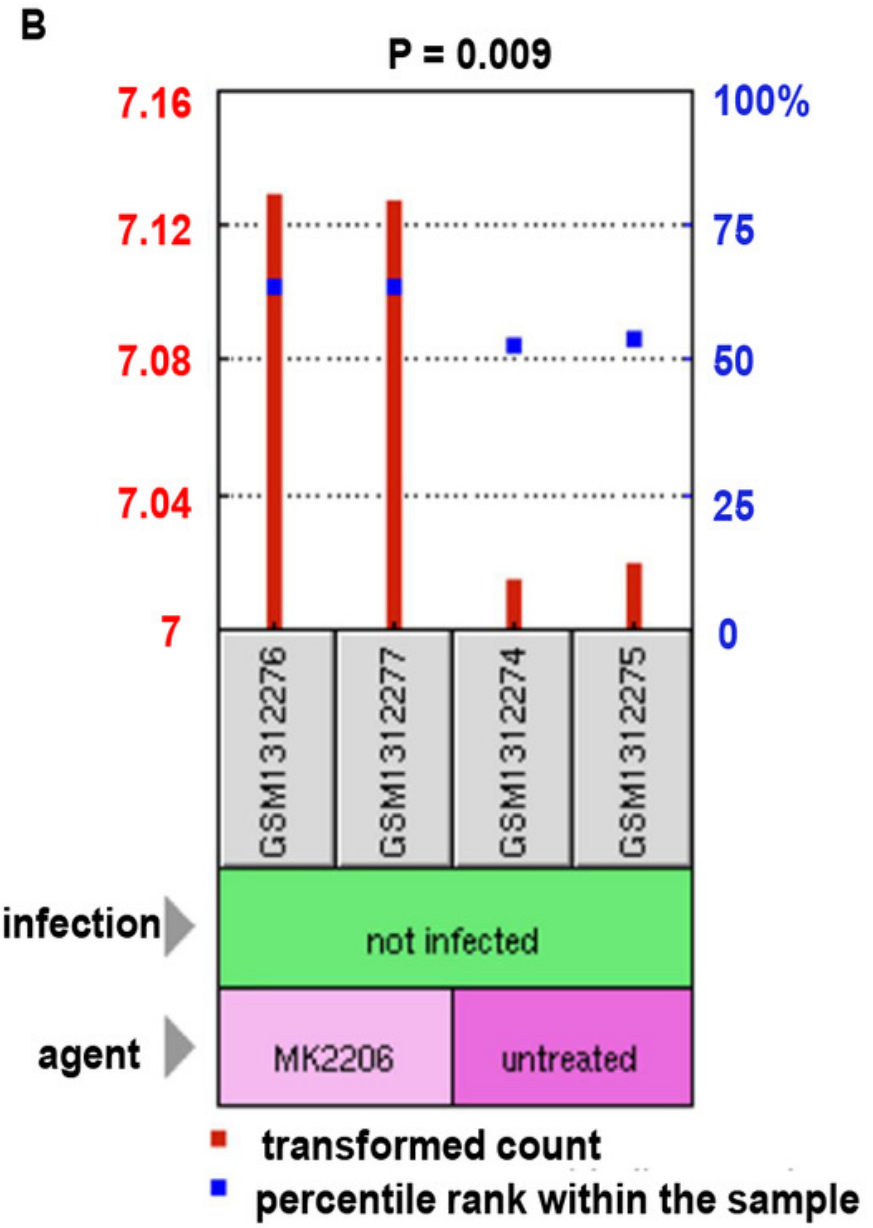


Figure 4

The relationship between NTRK2 methylation and the clinical characteristics of LUAD patients.

(A) Global NTRK2 methylation in LUAD samples compared with the normal samples analyzed by MethHC database. (B) The association between global NTRK2 methylation and its expression in LUAD samples using the MethHC database. (C) The impact of the methylation site cg03628748 in NTRK2 on OS in LUAD patients as analyzed by the MethSurv web tool.

A

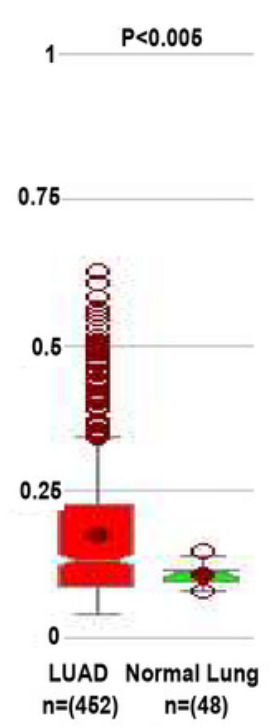

B

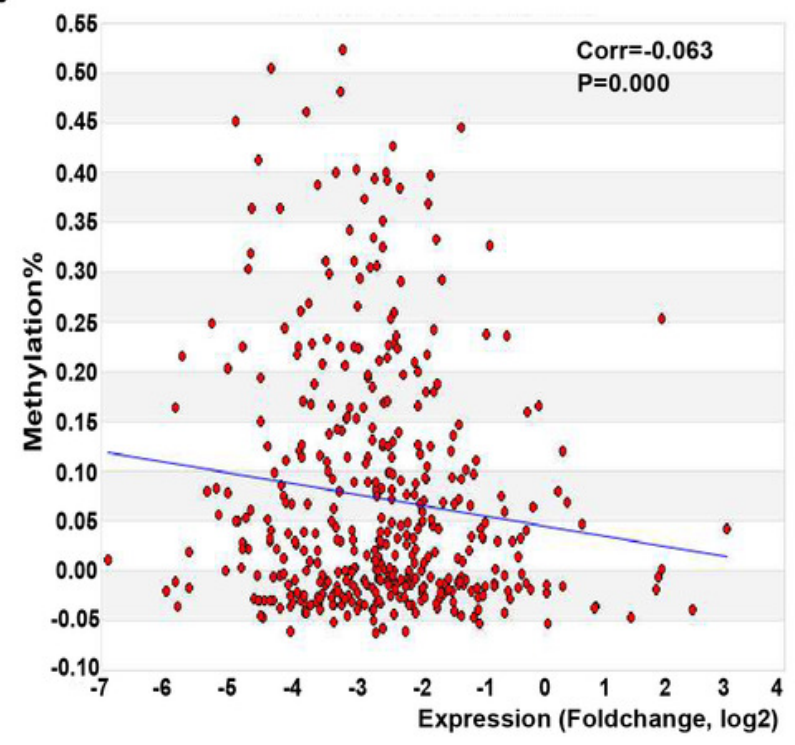

C

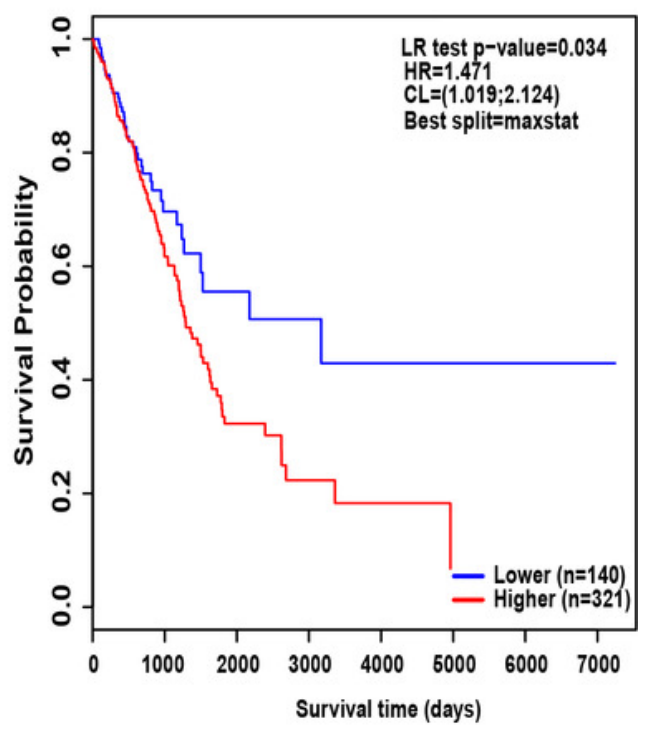


Figure 5

Functional enrichment analysis of NTRK2-associated co-DEGs in LUAD.

(A) The coexpression genes of NTRK2 were shown as volcano plot. (B) The PPI network of NTRK2-associated co-DEGs as completed by the STRING and Cytoscape software. (C-E) The GO analysis of NTRK2 associated co-DEGs including biological processes, cellular components and molecular function. 
A

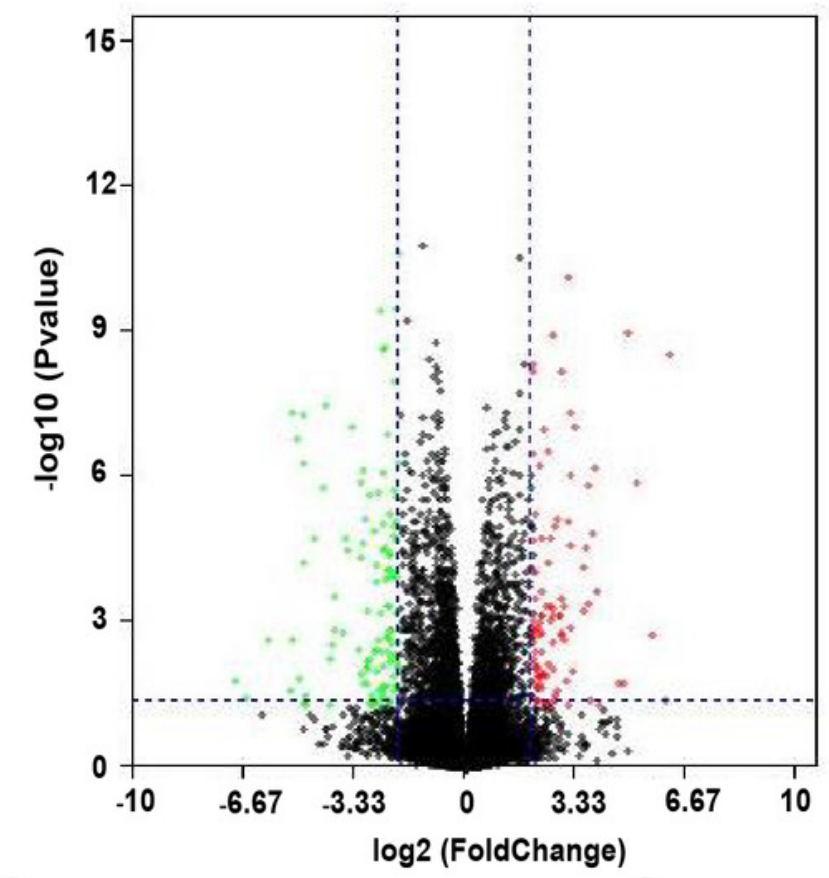

C

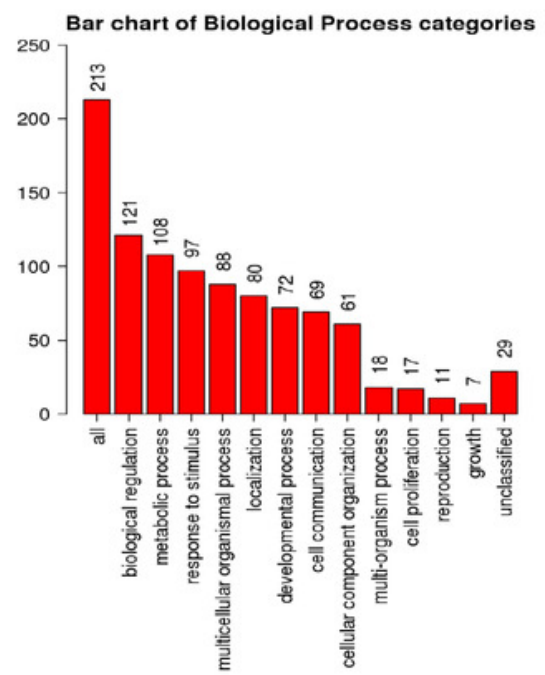

B

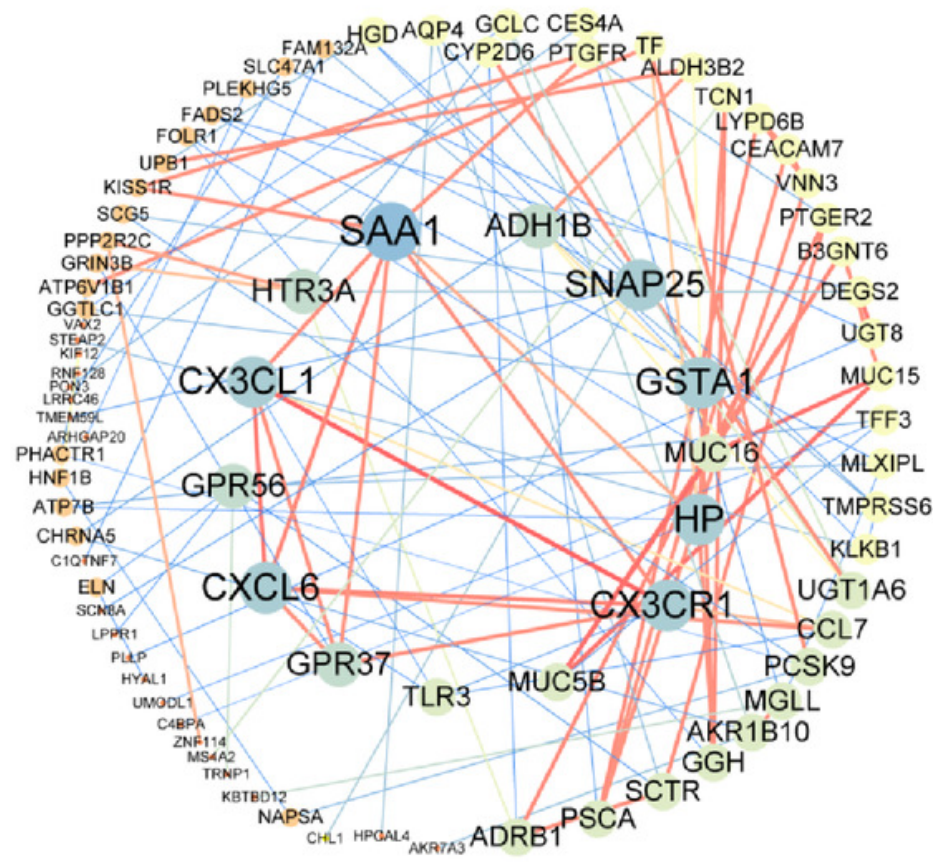

E

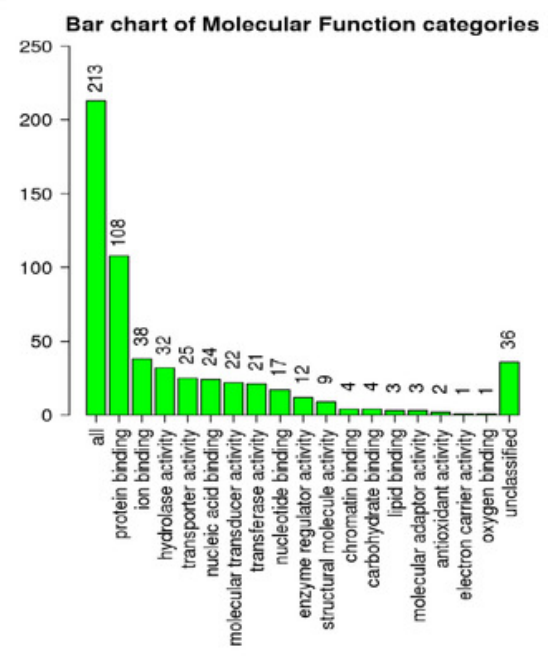




\section{Table $\mathbf{1}$ (on next page)}

The correlation between clinical characteristic parameters and the expression of NTRK2 in LUAD. 
1 Table 1:

2 The correlation between clinical characteristic parameters and the expression of NTRK2 in LUAD.

\begin{tabular}{|c|c|c|c|}
\hline Variables & Number & Mean \pm SD & $\mathbf{P}$ \\
\hline Gender & & & 0.007 \\
\hline Male & 179 & $5.25 \pm 1.90$ & \\
\hline Female & 212 & $5.78 \pm 1.95$ & \\
\hline Radiation therapy & & & 0.640 \\
\hline Yes & 6 & $5.12 \pm 1.31$ & \\
\hline No & 89 & $5.48 \pm 1.82$ & \\
\hline Kras mutation found & & & 0.454 \\
\hline Yes & 14 & $5.48 \pm 1.89$ & \\
\hline No & 34 & $5.88 \pm 1.54$ & \\
\hline Pathologic T & & & 0.021 \\
\hline $\mathrm{T} 1 / \mathrm{T} 1 \mathrm{a} / \mathrm{T} 1 \mathrm{~b}$ & 122 & $6.01 \pm 1.94$ & \\
\hline $\mathrm{T} 2 / \mathrm{T} 2 \mathrm{a} / \mathrm{T} 2 \mathrm{~b}$ & 218 & $5.35 \pm 2.01$ & \\
\hline $\mathrm{T} 3$ & 34 & $5.29 \pm 1.43$ & \\
\hline $\mathrm{T} 4$ & 15 & $5.09 \pm 1.52$ & \\
\hline $\mathrm{TX}$ & 2 & $4.36 \pm 1.56$ & \\
\hline Pathologic N & & & 0.875 \\
\hline N0 & 252 & $5.52 \pm 1.86$ & \\
\hline N1 & 71 & $5.59 \pm 1.89$ & \\
\hline $\mathrm{N} 2$ & 61 & $5.55 \pm 2.32$ & \\
\hline NX & 5 & $4.85 \pm 2.40$ & \\
\hline Pathologic M & & & 0.006 \\
\hline M0 & 255 & $5.38 \pm 1.85$ & \\
\hline $\mathrm{M} 1 / \mathrm{M} 1 \mathrm{a} / \mathrm{M} 1 \mathrm{~b}$ & 16 & $4.86 \pm 2.20$ & \\
\hline MX & 117 & $5.99 \pm 2.04$ & \\
\hline Pathologic stage & & & 0.471 \\
\hline Stage I/IA/IB & 211 & $5.63 \pm 1.89$ & \\
\hline Stage IIA/IIB & 94 & $5.45 \pm 1.78$ & \\
\hline Stage IIIA/IIIB & 68 & $5.52 \pm 2.25$ & \\
\hline Stage IV & 17 & $4.89 \pm 2.14$ & \\
\hline Race & & & 0.758 \\
\hline White & 314 & $5.60 \pm 1.92$ & \\
\hline Black or African American & 23 & $5.41 \pm 2.26$ & \\
\hline Asian & 5 & $5.07 \pm 1.12$ & \\
\hline Tobacco smoking history & & & 0.097 \\
\hline Current reformed smoker for $>15$ years & 94 & $5.84 \pm 2.06$ & \\
\hline Current reformed smoker for $<$ or $=15$ years & 131 & $5.38 \pm 1.95$ & \\
\hline Current reformed smoker, duration not specified & 2 & $5.15 \pm 1.34$ & \\
\hline Lifelong non-smoker & 61 & $5.91 \pm 1.75$ & \\
\hline
\end{tabular}




\begin{tabular}{lccc}
\hline $\begin{array}{l}\text { Current smoker } \\
\text { Age at initial pathologic diagnosis }\end{array}$ & 91 & $5.21 \pm 1.97$ & \\
$\leq 60$ & 125 & $5.25 \pm 1.86$ & \\
$>60$ & 248 & $5.69 \pm 1.94$ & \\
EGFR mutation result & & & 0.303 \\
Exon 19 Deletion & 7 & $5.07 \pm 1.26$ & \\
L858R & 3 & $6.47 \pm 1.00$ & \\
Other & 9 & $5.94 \pm 1.58$ & \\
\hline
\end{tabular}

3

4 


\section{Table 2 (on next page)}

The correlation between clinical characteristics of patients and the methylation site cg03628748 in NTRK2 in LUAD. 
1 Table 2:

2 The correlation between clinical characteristics of patients and the methylation site cg03628748 in NTRK2 in 3 LUAD.

\begin{tabular}{|c|c|c|c|}
\hline Variables & Number & Mean \pm SD & $\mathbf{P}$ \\
\hline Gender & & & 0.123 \\
\hline Male & 189 & $0.32 \pm 0.14$ & \\
\hline Female & 219 & $0.30 \pm 0.14$ & \\
\hline Radiation therapy & & & 0.112 \\
\hline Yes & 7 & $0.22 \pm 0.097$ & \\
\hline No & 96 & $0.31 \pm 0.14$ & \\
\hline Kras mutation found & & & 0.038 \\
\hline Yes & 16 & $0.38 \pm 0.18$ & \\
\hline No & 34 & $0.28 \pm 0.13$ & \\
\hline Pathologic T & & & 0.000 \\
\hline $\mathrm{T} 1 / \mathrm{T} 1 \mathrm{a} / \mathrm{T} 1 \mathrm{~b}$ & 127 & $0.26 \pm 0.11$ & \\
\hline $\mathrm{T} 2 / \mathrm{T} 2 \mathrm{a} / \mathrm{T} 2 \mathrm{~b}$ & 227 & $0.32 \pm 0.14$ & \\
\hline $\mathrm{T} 3$ & 36 & $0.35 \pm 0.16$ & \\
\hline $\mathrm{T} 4$ & 15 & $0.30 \pm 0.15$ & \\
\hline $\mathrm{TX}$ & 3 & $0.23 \pm 0.17$ & \\
\hline Pathologic N & & & 0.464 \\
\hline N0 & 261 & $0.31 \pm 0.14$ & \\
\hline N1 & 75 & $0.30 \pm 0.13$ & \\
\hline N2 & 62 & $0.30 \pm 0.14$ & \\
\hline NX & 8 & $0.24 \pm 0.11$ & \\
\hline Pathologic M & & & 0.183 \\
\hline M0 & 264 & $0.31 \pm 0.14$ & \\
\hline $\mathrm{M} 1 / \mathrm{M} 1 \mathrm{a} / \mathrm{M} 1 \mathrm{~b}$ & 17 & $0.27 \pm 0.16$ & \\
\hline MX & 123 & $0.29 \pm 0.13$ & \\
\hline Pathologic stage & & & 0.746 \\
\hline Stage I/IA/IB & 218 & $0.31 \pm 0.14$ & \\
\hline Stage IIA/IIB & 102 & $0.31 \pm 0.13$ & \\
\hline Stage IIIA/IIIB & 68 & $0.31 \pm 0.14$ & \\
\hline Stage IV & 19 & $0.27 \pm 0.16$ & \\
\hline Race & & & 0.214 \\
\hline White & 325 & $0.30 \pm 0.13$ & \\
\hline Blackor African American & 29 & $0.27 \pm 0.12$ & \\
\hline Asian & 5 & $0.37 \pm 0.14$ & \\
\hline Tobacco smoking history & & & 0.075 \\
\hline Current reformed smoker for $>15$ years & 101 & $0.31 \pm 0.15$ & \\
\hline Current reformed smoker for $<$ or $=15$ years & 135 & $0.32 \pm 0.14$ & \\
\hline Current reformed smoker, duration not specified & 2 & $0.39 \pm 0.022$ & \\
\hline
\end{tabular}




\begin{tabular}{lccc}
\hline Lifelong non-smoker & 62 & $0.26 \pm 0.12$ & \\
Current smoker & 96 & $0.31 \pm 0.14$ & \\
Age at initial pathologic diagnosis & & & 0.644 \\
$\leq 60$ & 131 & $0.30 \pm 0.14$ & \\
$>60$ & 259 & $0.31 \pm 0.13$ & \\
Residual tumor & & & 0.542 \\
RX & 16 & $0.31 \pm 0.15$ & \\
R0 & 271 & $0.31 \pm 0.14$ & \\
R1 & 10 & $0.26 \pm 0.093$ & \\
EGFR mutation result & & & 0.082 \\
Exon 19 Deletion & 7 & $0.18 \pm 0.063$ & \\
L858R & 3 & $0.28 \pm 0.17$ & \\
Other & 9 & $0.33 \pm 0.13$ & \\
\hline
\end{tabular}

4 